Modern Physics Letters A

(C) World Scientific Publishing Company

\title{
FINAL STATE INTERACTIONS AND THE TRANSVERSE STRUCTURE OF THE PION
}

\author{
LEONARD GAMBERG ${ }^{1,2}$ and MARC SCHLEGEL ${ }^{3}$ \\ 1 Institute for Nuclear Theory, University of Washington \\ Seattle, Washington 98195-1550, USA \\ ${ }^{2}$ Division of Science, Penn State University-Berks \\ Reading, Pennsylvania 19083, USA \\ 3 Thomas Jefferson National Accelerator Facility, \\ Newport News, VA 23606, USA
}

Received (28 October 2009)

\begin{abstract}
In the factorized picture of semi-inclusive deep inelastic scattering the naive time reversalodd parton distributions exist by virtue of the gauge link which is intrinsic to their definition. The link structure describes initial/final-state interactions of the active parton due to soft gluon exchanges with the target remnant. Though these interactions are non-perturbative, calculations of final-state interaction have been performed in a perturbative one-gluon approximation. We include higher-order contributions by applying non-perturbative eikonal methods to calculate the Boer-Mulders function of the pion. Using this framework we explore under what conditions the Boer Mulders function can be described in terms of factorization of final state interactions and a spatial distortion.
\end{abstract}

Keywords: transverse momentum dependent parton distribution function (TMD); semiinclusive deeply inelastic scattering (SIDIS); single spin asymmetry (SSA)

13.88.+e, 13.85.Ni, 13.60.-r, 13.85.Qk

\section{T-odd PDFs, Gluonic Poles and The Lensing Function}

Over the past two decades the transverse partonic structure of hadrons has been the subject of a great deal of theoretical and experimental study. Central to these investigations are the early observations of large transverse single spin asymmetries (TSSAs) in inclusive hadron production from proton-proton scattering over a wide range of beam energies. ${ }^{1-4}$ Recently TSSAs have been observed in lepton-hadron semi-inclusive deep inelastic scattering (SIDIS) $)^{5-9}$ as well as in inclusive production of pseudo-scalar mesons from in proton-proton collisions. ${ }^{10-13}$ While the naive parton model predicts that transverse polarization effects are trvial in the helicity limit, ${ }^{14}$ Efremov and Teryaev demonstrated ${ }^{15,16}$ that soft gluonic and fermionic poles contribute to multiparton correlation functions resulting in non-trivial twistthree transverse polarization effects in this limit. ${ }^{17,18}$ In addition theoretical work on transversity ${ }^{19-21}$ indicates that transverse polarization effects can appear at leading twist. Two explanations to account for TSSAs in QCD have emerged which are 
based on the twist-three ${ }^{17,18}$ and twist-two ${ }^{21-25}$ approaches. Recently, a coherent picture has emerged which describes TSSAs in a kinematic regime where the two approaches are expected to have a common description. ${ }^{26-29}$

In the factorized picture of semi-inclusive deep inelastic scattering ${ }^{24,30}$ at small transverse momenta $P_{T} \sim k_{T}<<\sqrt{Q^{2}}$ the Sivers effect describes a transverse target spin- $S_{T}$ asymmetry through the "naive" T-odd structure, $\Delta f\left(x, \vec{k}_{T}\right) \sim$ $S_{T} \cdot\left(P \times \vec{k}_{T}\right) f_{1 T}^{\perp}\left(x, k_{T}^{2}\right) \cdot{ }^{22,31}$ For an unpolarized target with transversely polarized quarks- $s_{T}$, the Boer-Mulders function ${ }^{25}$ is $\Delta h\left(x, \vec{k}_{T}\right) \sim s_{T} \cdot\left(P \times \vec{k}_{T}\right) h_{1}^{\perp}\left(x, k_{T}^{2}\right)$. Many studies have been performed to model the T-odd PDFs in terms of the FSIs where soft gluon rescattering effects are approximated by perturbative one-gluon exchange. ${ }^{32-41}$ We improve this approximation by applying non-perturbative eikonal methods to calculate higher-order gluonic contributions from the gauge link in the spectator framework. ${ }^{42}$ In the context of these higher order contributions we perform a quantitative study of approximate relations between TMDs and GPDs. In particular, we explore under what conditions the T-odd PDFs can be described via factorization of FSI and spatial distortion of impact parameter space PDFs. ${ }^{43}$ While such relations are fulfilled from lowest order contributions in field-theoretical spectator models ${ }^{44,45}$ a model-independent analysis of generalized parton correlation functions $(\mathrm{GPCFs})^{46}$ indicates that the Sivers function and the helicity flip GPD $E$ are projected from independent GPCFs. A similar result holds for the Boer-Mulders function for a spin zero target. ${ }^{47}$ From phenomenology, however it essentially unknown whether the proposed factorization is a good approximation. Here we focus on the transverse structure of the pion through the impact parameter GPD and the Boer Mulders function for which very little know. Recent lattice calculations indicate that the spatial asymmetry of transversely polarized quarks in the pion is quite similar in magnitude to that of quarks in the nucleon. ${ }^{48}$

The field-theoretical definition of transverse-momentum dependent (TMD) parton distributions in terms of hadronic matrix elements of quark operators for spin1/2 hadron with momentum $P$ and spin $S$ was presented in Refs. ${ }^{24,29,49}$ It is straightforward to obtain the TMDs for a spin-0 hadron from that. One encounters two leading twist TMDs for a pion, the distribution for unpolarized quarks $f_{1}$, and the distribution of transversely polarized quarks $h_{1}^{\perp}$, the Boer-Mulders function. Adopting the infinite-momentum frame where the hadron moves relativistically along the positive $z$-axis such that the target momentum $P$ has a large plus component $P^{+}$and no transverse component the Boer-Mulders function, defined in SIDIS is

$$
\frac{\epsilon_{T}^{i j} k_{T}^{j} h_{1}^{\perp}\left(x, \vec{k}_{T}^{2}\right)}{m_{\pi}}=\int \frac{d z^{-} d^{2} z_{T}}{4(2 \pi)^{3}} \mathrm{e}^{i x P^{+} z^{-}-i \vec{k}_{T} \cdot \vec{z}}\left\langle P\left|\bar{q}_{j}(0)[0 ; \infty n] i \sigma^{i+} \gamma_{5}\left[\infty n+z_{T} ; z\right] q_{i}(z)\right| P\right\rangle .
$$

The light-like vector $n$ represents a specific direction on the light-cone $n^{\mu}=(1,0,0)$ where we define the light cone components of a 4 -vector $a^{ \pm}=1 / \sqrt{2}\left(a^{0} \pm a^{3}\right)$, $a^{\mu}=\left(a^{-}, a^{+}, a^{\perp}\right) .[x ; y]$ denotes a gauge link operator connecting the two locations $x$ and $y$. We work in a covariant gauge where a transverse gauge link at light-cone 
infinity is negligible. The gauge link in (1) is interpreted physically as FSIs of the active quark with the target remnants ${ }^{32,50}$ and is necessary for "naive" time-reversal odd (T-odd) TMDs ${ }^{22,25,31}$ to exist. ${ }^{50}$ The Boer-Mulders function appears in the factorized description of semi-inclusive processes such as SIDIS ${ }^{24-26,30,51-56}$ in terms of the first $k_{T}$-moment, $2 m_{\pi}^{2} h_{1}^{\perp(1)}(x)=\int d^{2} k_{T} \vec{k}_{T}^{2} h_{1}^{\perp}\left(x, \vec{k}_{T}^{2}\right)$. Transforming the two pion states in Eq. (11) into a mixed coordinate-momentum representation specified by the impact parameter $b_{T}{ }^{45,57}$ results in an impact parameter representation for the gluonic pole matrix element ${ }^{26}\left\langle k_{T}\right\rangle(x)=m_{\pi} h_{1}^{\perp(1)}(x)$,

$$
\left\langle k_{T}\right\rangle(x)=\int d^{2} b_{T} \frac{d z^{-}}{4(2 \pi)} \mathrm{e}^{i x P^{+} z^{-}}\left\langle P^{+}, \overrightarrow{0}_{T}\left|\bar{q}\left(z_{1}\right)\left[z_{1} ; z_{2}\right] I^{i}\left(z_{2}\right) \sigma^{i+} q\left(z_{2}\right)\right| P^{+}, \overrightarrow{0}_{T}\right\rangle .
$$

Here, the impact parameter $b_{T}$ is hidden in the arguments of the quark fields, $z_{1 / 2}^{\mu}=\mp \frac{z^{-}}{2} n^{\mu}+b_{T}^{\mu}$ and $b_{T}^{\mu}=\left(0, b_{T}^{1}, b_{T}^{2}, 0\right)$. The operator $I^{i}$ originates from the time-reversal behavior of the FSIs written in terms of the gauge link in (11) and the field strength tensor $F^{\mu \nu}$,

$$
2 I^{i}\left(z_{2}\right)=\int d y^{-}\left[z_{2} ; y\right] g F^{+i}(y)\left[y ; z_{2}\right],
$$

with $y^{\mu}=y^{-} n^{\mu}+b_{T}^{\mu}$.

Turning our attention to GPDs of a pion, they are represented by an off-diagonal matrix element of a quark-quark operator defined on the light-cone. ${ }^{58-60}$ One encounters two leading twist GPDs for a pion, a chirally-even GPD $F_{1}^{\pi}$ and the chiral odd GPDs $H_{1}^{\pi} \cdot{ }^{47}$ We use the symmetric conventions for the kinematics for GPDs, ${ }^{58}$ $P=\frac{1}{2}\left(p+p^{\prime}\right)$ and $\Delta=p^{\prime}-p$ where $\Delta^{+}=-2 \xi P^{+}$, and $t=\Delta^{2}$. The impact parameter GPDs are obtained from the ordinary GPDs via a Fourier-transform of the transverse momentum transfer $\vec{\Delta}_{T}$ at zero skewness $\xi=0$. The chirally-odd impact parameter GPD $\mathcal{H}_{1}^{\pi}$ is expressed as

$$
\int \frac{d z^{-}}{2(2 \pi)} \mathrm{e}^{i x P^{+} z^{-}}\left\langle P^{+}, \overrightarrow{0}_{T}\left|\bar{q}\left(z_{1}\right)\left[z_{1} ; z_{2}\right] \sigma^{+i} q\left(z_{2}\right)\right| P^{+}, \overrightarrow{0}_{T}\right\rangle=\frac{2 b_{T}^{i}}{m_{\pi}} \frac{\partial}{\partial \vec{b}_{T}^{2}} \mathcal{H}_{1}^{\pi}\left(x, \vec{b}_{T}^{2}\right) .
$$

$\mathcal{H}_{1}^{\pi}$ describes how transversely polarized quarks are distributed in a plane transverse to the direction of motion. This distribution represents transverse space distortion due to spin-orbit correlations. ${ }^{48,61,62}$ A comparison of the first moment of the Boer Mulders function (1) and the impact parameter GPD $\mathcal{H}_{1}^{\pi}$ reveals that they differ by the operator $I^{i}$ which represents the FSIs. In various model calculations, the FSIs are approximated such that ${ }^{44,45,57,63}$ the two effects of a distortion of the transverse space parton distribution and the FSIs factorize resulting in the quantitative relation

$$
m_{\pi} \epsilon_{T}^{i j} h_{1}^{\perp(1)}(x) \simeq \int d^{2} b_{T} \mathcal{I}^{j}\left(x, \vec{b}_{T}\right) \frac{\epsilon_{T}^{i l} b_{T}^{l}}{m_{\pi}} \frac{\partial}{\partial \vec{b}_{T}^{2}} \mathcal{H}_{1}^{\pi}\left(x, \vec{b}_{T}^{2}\right),
$$

where $\mathcal{I}$ is the so-called "quantum chromodynamic lensing function". ${ }^{57}$ This factorization doesn't hold in general. ${ }^{47,64}$ For example, this relation breaks down when the quark fields and the operator $I$ in (1) "interact" via quantum fluctuations (because they are interacting Heisenberg operators). However, it unknown if (4) is a 


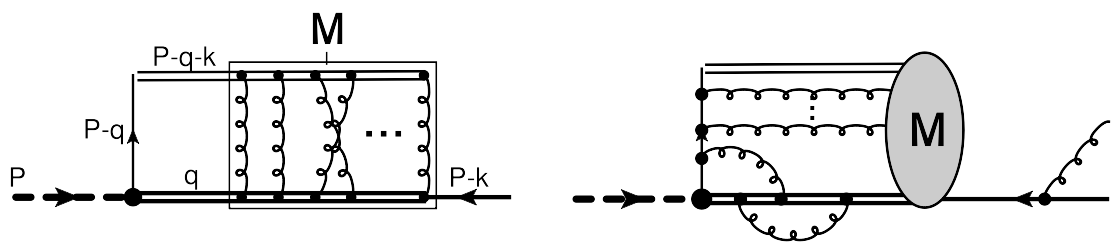

Fig. 1. The amplitude $W$ including FSIs between re-scattered eikonalized quark and antiquark. The FSIs are described by a non-perturbative scattering amplitude $M$ that is calculated in a generalized ladder approximation. Gluon interactions as shown in the second diagram are not taken into account (see text).

good phenomenological approximation. We estimate the size of the lensing function using non-perturbative eikonal methods ${ }^{65,66}$ to calculate higher-order gluon contributions from the gauge link. Up till now the relation (4) was used to predict the sign of T-odd TMDs in conjunction with numbers for the $u$ - and $d$-quark contributions to the anomalous magnetic moment of the nucleon and the assumption that final state interactions are attractive. ${ }^{61}$

\section{TMD - GPD Relation for a Pion the Spectator Framework}

We focus our attention in the following on a pion in a valence quark-type configuration that one would expect for relatively large Bjorken $x$. Thus, we only take valence quark wave functions into account and neglect higher Fock states. Assuming an antiquark spectator one can express the pion Boer-Mulders function (1) as

$$
\epsilon_{T}^{i j} k_{T}^{j} h_{1}^{\perp}\left(x, \vec{k}_{T}^{2}\right)=\frac{m_{\pi}}{8(2 \pi)^{3}(1-x) P^{+}} \sum_{\sigma, d} \bar{W} i \sigma^{i+} \gamma_{5} W,
$$

where $W_{i}^{\alpha, \delta}(P, k ; \sigma)=\left\langle P-k, \sigma, \delta\left|[\infty n ; 0]^{\alpha \beta} q_{i}^{\beta}(0)\right| P\right\rangle$ and $\sigma$ and $\delta$ represent the helicity and color of the intermediate spectator antiquark. We model the matrix element for $W$ by a diagram shown in Fig. 1, where the final state interactions generated by the gauge link in $W$ - are described by a non-perturbative amputated scattering amplitude $(M)_{\gamma \delta}^{\alpha \beta}$ with $\beta, \alpha(\gamma, \delta)$ color indices of incoming and outgoing quark (anti-quark), respectively. We neglect classes of gluon exchanges in the second diagram in Fig. 1 1 represented by the gluon rungs since they would be attributed to the "interaction" between the quark fields and the operator $I$ in (1). They lead to terms which break the relation (4). We also neglect real gluon emission and (self)interactions of quark and antiquark lines the second diagram in Fig. 1 since they represent radiative corrections of the GPD and are effectively modeled in terms of spectator masses and phenomenological vertex functions. The pion-quark vertex is governed by the interaction Lagrangian $\mathcal{L}=-g_{\pi} / \sqrt{N_{c}} \delta^{\alpha \beta} \bar{q}^{\alpha} \gamma_{5} \vec{\tau} \cdot \vec{\varphi} q^{\beta}$ and we allow the coupling constant $g_{\pi}$ to depend on the momentum of the active quark in order to account for the compositeness of the hadron and to suppress large quark virtualities. Phenomenological vertex functions in connection with spectator models have been used frequently in the literature. ${ }^{40,41,67}$ Applying the Feynman rules it is 
then possible to find an expression for the matrix element $W$ from the first diagram in Fig. 11 We find

$$
\begin{gathered}
W_{i, \sigma}^{\alpha \beta}(P, k)=\frac{-i \tau}{\sqrt{N_{c}}}\left[\delta^{\alpha \beta} g_{\pi}\left(k^{2}\right) \frac{\left[\left(\not k+m_{q}\right) v\left(P_{s}, \sigma\right)\right]_{i}}{k^{2}-m_{q}^{2}+i 0}-\int \frac{d^{4} q}{(2 \pi)^{4}}\right. \\
\left.\frac{g_{\pi}\left((P-q)^{2}\right)\left[\left(\not P-\not 1+m_{q}\right) \gamma_{5}\left(-\not h+m_{s}\right)(M)_{\delta \beta}^{\alpha \delta}\left(q, P_{s}\right) v\left(P_{s}, \sigma\right)\right]_{i}}{\left[n \cdot\left(P_{s}-q\right)+i 0\right]\left[(P-q)^{2}-m_{q}^{2}+i 0\right]\left[q^{2}-m_{s}^{2}+i 0\right]}\right],
\end{gathered}
$$

where $P_{s} \equiv P-k$ is the spectator momentum. The first term in (6) represents the contribution without final state interactions while the second term corresponds to the first diagram in Fig. 1. We then express the FSIs through the amputated quark - anti-quark scattering amplitude $M$. Here both incoming quark and antiquark are subject to the eikonal approximation (see, e.g. Ref. 68 and references therein). While the active quark undergoes a natural eikonalization for a massless fermion since it represents the gauge link contribution, the eikonalization for a massive spectator fermion is a simplification that can be justified by the physical picture of partons in an infinite momentum frame. The eikonalization of a massive fermion can be traced back to the Nordsieck-Bloch approximation ${ }^{69}$ which describes a highly energetic helicity conserving fermion undergoing multiple scattering undergoing very small momentum transfer. In this approximation the Dirac vertex structure, $\bar{u}\left(p_{1}\right) \gamma^{\mu} u\left(p_{2}\right) \sim p_{\mu} / m$ where $\left(p_{1}+p_{2}\right) / 2 \equiv p$. For a massive anti-fermion one identifies the velocity $v^{\mu}=-p^{\mu} / m$, and the numerator of a fermion propagator becomes $i(-\not p+m) \rightarrow i(-\bar{n} \cdot p+m)$.

We proceed by performing a contour-integration of the light-cone loopmomentum, $q^{-}$in Eq. (6) . In doing so we only consider poles which originate from the denominators in (6). This assumes that the scattering amplitude $M$ does not contain poles in $q^{-}$. This assumption is not necessarily true even for a one gluon exchange approximation. As shown in Refs. $70,40, q^{-}$poles appearing in $M$ are related to light-cone divergences that may be regulated by choosing a slightly off-light like vector $n$. Performing the contour integration under the described assumptions fixes the momentum $q^{-}$of the anti-quark in the loop in (6) to $q^{-}=\left(\vec{q}_{T}^{2}+m_{s}^{2}\right) / 2 q^{+}$. The eikonal propagator can be split into a real and imaginary part via the usual principle value prescription $1 /(x+i 0)=\mathcal{P}(1 / x)-i \pi \delta(x)$. It has been argued ${ }^{45}$ that only the imaginary part contributes to the relation (4) as it forces the antiquark momentum $q$ to be on the mass shell. Thus, the imaginary part of the eikonal propagator corresponds to a cut of the first diagram in Fig. 1 before the final-state interactions take place, whereas the real part given by the principle value is attributed to terms that break the relation (4).

After performing these integrations we use (6) to calculate the pion BoerMulders function via (5). The pion-quark-antiquark vertex is

$$
g_{\pi}\left(k^{2}\right)=g_{\pi} \frac{\left(-\Lambda^{2}\right)^{n-1}}{(n-1) !} \partial_{\Lambda^{2}}^{n-1} \frac{\left(k^{2}-m_{q}^{2}\right) f\left(k^{2}\right)}{k^{2}-\Lambda^{2}+i 0},
$$


where the function $f$ is a homogeneous function of the quark virtuality. We choose it to be a Gaussian $\exp \left[-\lambda^{2}\left|k^{2}\right|\right] .^{40}$ Inserting (6) into (5) yields the following expression for the Boer-Mulders function,

$$
\begin{aligned}
& \epsilon_{T}^{i j} k_{T}^{j} h_{1}^{\perp}\left(x, \vec{k}_{T}^{2}\right)=\frac{2 g_{\pi}^{2} m_{\pi}}{(2 \pi)^{3} \Lambda^{2}}\left(x m_{s}+(1-x) m_{q}\right)\left((1-x) \Lambda^{2}\right)^{2 n-1} \\
& \times \int \frac{d^{2} q_{T}}{(2 \pi)^{2}} \frac{d^{2} p_{T}}{(2 \pi)^{2}} \epsilon_{T}^{j i}\left(q_{T}^{j}-p_{T}^{j}\right) \frac{\mathrm{e}^{-\frac{2 \lambda^{2}}{1-x}\left(x m_{s}^{2}-x(1-x) m_{\pi}^{2}\right)} \mathrm{e}^{-\frac{\lambda^{2}}{1-x}\left(\tilde{\mathrm{q}}_{\mathrm{T}}^{2}+\tilde{\mathrm{p}}_{\mathrm{T}}^{2}\right)}}{\left[\vec{q}_{T}^{2}+\tilde{\Lambda}^{2}(x)\right]^{n}\left[\vec{p}_{T}^{2}+\tilde{\Lambda}^{2}(x)\right]^{n}} \mathcal{F}\left[\begin{array}{l}
\mathrm{eik} \\
\end{array},\right.
\end{aligned}
$$

where

$\mathcal{F}\left[\bar{M}^{\mathrm{eik}}\right] \equiv\left(\Im\left[\overline{\mathcal{M}}^{\mathrm{eik}}\right]\right)_{\delta \beta}^{\alpha \delta}\left(\vec{k}_{T}+\vec{q}_{T}\right)\left((2 \pi)^{2} \delta^{\alpha \beta} \delta^{(2)}\left(\vec{p}_{T}+\vec{k}_{T}\right)+\left(\Re\left[\bar{M}^{\mathrm{eik}}\right]\right)_{\gamma \alpha}^{\beta \gamma}\left(\vec{k}_{T}+\vec{p}_{T}\right)\right)$

with $\tilde{\Lambda}^{2}(x)=x m_{s}^{2}-x(1-x) M^{2}+(1-x) \Lambda^{2}$. This result already anticipates an eikonal form for the scattering amplitude $\bar{M}\left(x, \vec{k}_{T}, \vec{q}_{T}\right) \rightarrow \bar{M}^{\text {eik }}\left(\left|\vec{q}_{T}+\vec{k}_{T}\right|\right)$ which we exploit to simplify the expression and show a relation to the chirally-odd GPD $H_{1}^{\pi}$. A calculation for the GPD $H_{1}^{\pi}$ for an antiquark spectator can be found in Ref. 47, which we generalize with the vertex function $g_{\pi}\left(k^{2}\right)$

$$
\begin{aligned}
& H_{1}^{\pi}\left(x, 0,-\vec{\Delta}_{T}^{2}\right)=\frac{-g_{\pi}^{2} m_{\pi}}{2(2 \pi)^{3} \Lambda^{2}}\left(x m_{s}+(1-x) m_{q}\right)\left(\frac{(1-x) \Lambda^{2}}{\vec{D}_{T}^{2}+\tilde{\Lambda}^{2}(x)}\right)^{2 n-1} \\
& \times \int_{0}^{2 \pi} d \varphi \int_{0}^{1} d z \frac{z^{2 n-2} \mathrm{e}^{2 \lambda^{2} \Lambda^{2}} \mathrm{e}^{-\frac{2 \lambda^{2}\left(\vec{D}_{T}^{2}+\tilde{\Lambda}^{2}(x)\right)}{(1-x) z}}}{\left[1-4 z(1-z) \frac{\vec{D}_{T}^{2}}{\vec{D}_{T}^{2}+\tilde{\Lambda}^{2}(x)} \cos ^{2} \varphi\right]^{n}}
\end{aligned}
$$

where $\vec{D}_{T}^{2}=\frac{1}{4}(1-x)^{2} \vec{\Delta}_{T}^{2}$. Weighting (8) with a transverse quark vector $k_{T}^{i}$ and integrating both sides over $k_{T}$ we readily obtain the relation

$$
m_{\pi}^{2} h_{1}^{\perp(1)}(x)=\int \frac{d^{2} q_{T}}{2(2 \pi)^{2}} \vec{q}_{T} \cdot \overrightarrow{\mathcal{I}}\left(x, \vec{q}_{T}\right) H_{1}^{\pi}\left(x, 0,-\left(\frac{\vec{q}_{T}}{1-x}\right)^{2}\right) .
$$

The function $\mathcal{I}^{i}$ can be expressed in terms of the real and imaginary part of the scattering amplitude $\bar{M}$,

$$
\begin{gathered}
\mathcal{I}^{i}\left(x, \vec{q}_{T}\right)=\frac{1}{N_{c}} \int \frac{d^{2} p_{T}}{(2 \pi)^{2}}\left(2 p_{T}-q_{T}\right)^{i}\left(\Im\left[\bar{M}^{\mathrm{eik}}\right]\right)_{\delta \beta}^{\alpha \delta}\left(\left|\vec{p}_{T}\right|\right) \\
\left((2 \pi)^{2} \delta^{\alpha \beta} \delta^{(2)}\left(\vec{p}_{T}-\vec{q}_{T}\right)+\left(\Re\left[\bar{M}^{\mathrm{eik}}\right]\right)_{\gamma \alpha}^{\beta \gamma}\left(\left|\vec{p}_{T}-\vec{q}_{T}\right|\right)\right) .
\end{gathered}
$$

In order to derive the relation (4) one transforms Eq. (11) into the impact parameter space via a Fourier transform. The lensing function in the impact parameter space then reads,

$$
\mathcal{I}^{i}\left(x, \vec{b}_{T}\right)=i(1-x) \int \frac{d^{2} q_{T}}{(2 \pi)^{2}} \mathrm{e}^{i \frac{\vec{q}_{T} \cdot \vec{b}_{T}}{1-x}} I^{i}\left(x, \vec{q}_{T}\right)
$$




\section{The Lensing and Boer Mulders Function in Relativistic Eikonal Approximation}

In order to calculate the $\mathbf{2} \rightarrow \mathbf{2}$ scattering amplitude $M$ (needed for (12)) we use functional methods to incorporate the color degrees of freedom in the eikonal limit when soft gauge bosons couple to highly energetic particles on the light cone. Here we summarize implementation of the color structure in the calculation of $M$ while the details of the functional approach can be found in a forthcoming publication. Work in this direction was carried out in Refs. 71, 66, 72. After some functional manipulations of the scattering amplitude, $M$ can be expressed in terms of quarkand antiquark-propagators that are linked together by soft colored gluons. The amplitude $M$ reduces to a simple, gauge-invariant expression ${ }^{66}$

$$
\begin{aligned}
& \left(M^{\text {eik }}\right)_{\delta \beta}^{\alpha \delta}\left(x,\left|\vec{q}_{T}+\vec{k}_{T}\right|\right)=\frac{(1-x) P^{+}}{m_{s}} \int d^{2} z_{T} \mathrm{e}^{-i \vec{z}_{T} \cdot\left(\vec{q}_{T}+\vec{k}_{T}\right)} \\
& \quad \times\left[\int d^{N_{c}^{2}-1} \alpha \int \frac{d^{N_{c}^{2}-1} u}{(2 \pi)^{N_{c}^{2}-1}} \mathrm{e}^{-i \alpha \cdot u}\left(\mathrm{e}^{i \chi\left(\left|\vec{z}_{T}\right|\right) t \cdot \alpha}\right)_{\alpha \delta}\left(\mathrm{e}^{i t \cdot u}\right)_{\delta \beta}-\delta_{\alpha \beta}\right] .
\end{aligned}
$$

In Eq. (14) the $N_{c}^{2}-1$ dimensional integrals over the color parameters results from auxiliary fields $\alpha^{a}(s)$ and $u^{a}(s)$ that were introduced in the functional formalism of Ref. 66 in order to decouple the gluon fields from the color matrices. The eikonal phase $\chi\left(\left|\vec{z}_{T}\right|\right)$ in Eq. (14) represents the arbitrary amount of soft gluon exchanges that are summed up into an exponential form and is expressed in terms of the gluon propagator in a covariant gauge,

$$
\chi\left(\left|\vec{z}_{T}\right|\right)=g^{2} \int_{-\infty}^{\infty} d \alpha \int_{-\infty}^{\infty} d \beta n^{\mu} \bar{n}^{\nu} \mathcal{D}_{\mu \nu}(z+\alpha n-\beta \bar{n}) .
$$

$\mathcal{D}$ denotes the gluon propagator, and $g$ the strong coupling. In this form the fourvector $v$ is related to the complementary light cone vector $\bar{n}, v=-\left((1-x) P^{+} / m_{s}\right) \bar{n}$, with $n \cdot \bar{n}=1$ and $\bar{n}^{2}=0$. We evaluate the color integrals by deriving a power series representation for the color function

$$
f_{\alpha \beta}(\chi) \equiv \int d^{N_{c}^{2}-1} \alpha \int \frac{d^{N_{c}^{2}-1} u}{(2 \pi)^{N_{c}^{2}-1}} \mathrm{e}^{-i \alpha \cdot u}\left(\mathrm{e}^{i \chi\left(\left|\vec{z}_{T}\right|\right) t \cdot \alpha}\right)_{\alpha \delta}\left(\mathrm{e}^{i t \cdot u}\right)_{\delta \beta}-\delta_{\alpha \beta} .
$$

After manipulating the exponentials in (16), rewriting the resulting factors as derivatives with respect to $u$, and performing an integration by parts we obtain the following power series representation for $f$,

$$
f_{\alpha \beta}(\chi)=\sum_{n=1}^{\infty} \frac{(i \chi)^{n}}{(n !)^{2}} \sum_{a_{1}=1}^{N_{c}^{2}-1} \ldots \sum_{a_{n}=1}^{N_{c}^{2}-1} \sum_{P_{n}}\left(t^{a_{1}} \ldots t^{a_{n}} t^{a_{P_{n}(1)}} \ldots t^{a_{P_{n}(n)}}\right)_{\alpha \beta},
$$

where $P_{n}$ represents the sum over all permutations of the set $\{1, \ldots, n\}$. If we had a direct ladder where gluons were not allowed to cross we would have only factors $\left(t^{a_{1}} \ldots t^{a_{n}} t^{a_{n}} \ldots t^{a_{1}}\right)_{\alpha \beta}=C_{F}^{n} \delta_{\alpha \beta}$ with $C_{F}=\frac{N_{c}^{2}-1}{2 N_{c}}$, and we could work in an Abelian theory with an effective replacement $\alpha \rightarrow C_{F} \alpha_{s}$ for the fine-structure constant. 

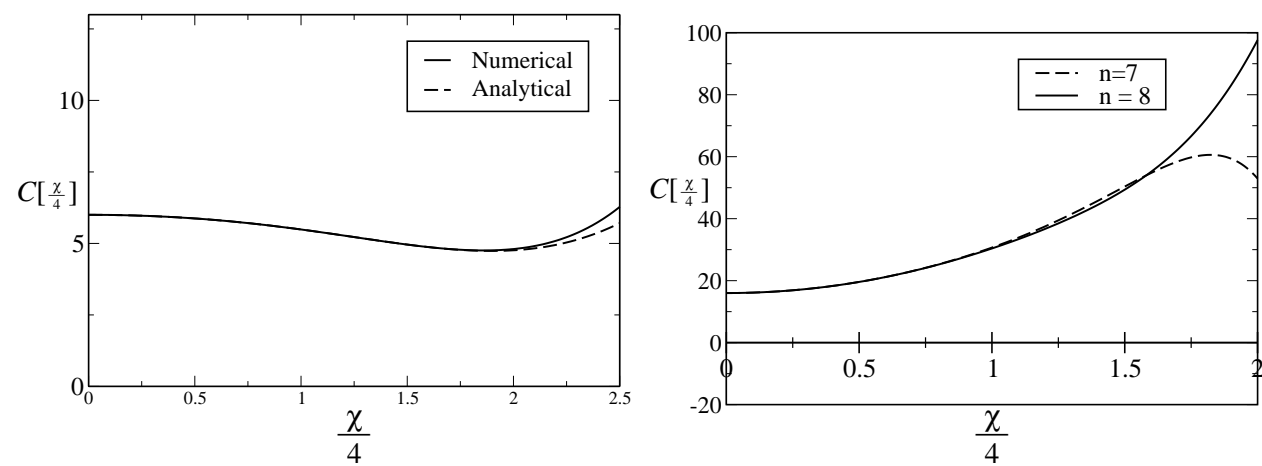

Fig. 2. The function $C\left[\frac{\chi}{4}\right]$ of Eq. (22) as a function of the eikonal amplitude $\frac{\chi}{4}$. Left: $S U(2)$, we compare the numerical result computed by means of Eq. (17) up to the order $n=8$ with the analytical result in Eq. (24). The numerical agrees with the analytical result up to $\frac{\chi}{4} \sim 2$. Right: $S U(3)$, we compare the numerical results for the orders $n=7,8$. The results are accurate up to $\frac{\chi}{4} \sim 1.5$.

Since we allow generalized ladders with crossed gluons we have to sum over all permutations in (17), and the simple replacement is not possible. In a large $N_{c}$ expansion the crossed gluons diagrams would be suppressed such that the direct ladder represents the leading order in $1 / N_{c}$. In an Abelian theory, the generating matrices $t$ reduce to identity and since we have $n$ ! permutations of the set $\{1, \ldots, n\}$, we recover the well-known Abelian result,

$$
f^{U(1)}(\chi)=\sum_{n=1}^{\infty} \frac{(i \chi)^{n}}{n !}=\mathrm{e}^{i \chi}-1 .
$$

For $N_{c}=2, t^{a}=\sigma^{a} / 2$ and we can calculate the integral (16) analytically by means of the relation $\left(\mathrm{e}^{i u \cdot \frac{\sigma}{2}}\right)_{\alpha \beta}=\delta_{\alpha \beta} \cos \left(\frac{|u|}{2}\right)+\frac{i \vec{\sigma}_{\alpha \beta} \cdot \vec{u}}{|u|} \sin \left(\frac{|u|}{2}\right)$. We obtain,

$$
f_{\alpha \beta}^{S U(2)}\left(\frac{\chi}{4}\right)=\delta_{\alpha \beta}\left(\cos \frac{\chi}{4}-\frac{\chi}{4} \sin \frac{\chi}{4}-1\right)+i \delta_{\alpha \beta}\left(2 \sin \frac{\chi}{4}+\frac{\chi}{4} \cos \frac{\chi}{4}\right) .
$$

We also calculate numerically the lowest coefficients in the power series (17), and they exactly agree with the coefficients in an expansion in $\chi$ of the analytical result (19). This serves as a check of both numerical and analytical approaches. For $N_{c}=3$, due to difficulty of integrating over the Haar measure we use the power series (17) to and obtain the following approximative color function which is valid if $a=\chi / 4$ is small,

$$
\begin{aligned}
& \Re\left[f_{\alpha \beta}^{S U(3)}\right](a)=\delta_{\alpha \beta}\left(-c_{2} a^{2}+c_{4} a^{4}-c_{6} a^{6}-c_{8} a^{8}+\ldots\right), \\
& \Im\left[f_{\alpha \beta}^{S U(3)}\right](a)=\delta_{\alpha \beta}\left(c_{1} a-c_{3} a^{3}+c_{5} a^{5}-c_{7} a^{7}+\ldots\right),
\end{aligned}
$$

with the numerical values $c_{1}=5.333, c_{2}=6.222, c_{3}=3.951, c_{4}=1.934, c_{5}=0.680$, $c_{6}=0.198, c_{7}=0.047, c_{8}=0.00967$. Working in coordinate space where we can express the lensing function directly in terms of the real and imaginary part of the 
color function $f$ which is itself a function of the eikonal phase $\chi$ Eq. (15), results in a lensing function of the form,

$$
\mathcal{I}^{i}\left(x, \vec{b}_{T}\right)=\frac{(1-x)}{2 N_{c}} \frac{b_{T}^{i}}{\left|\vec{b}_{T}\right|} \frac{\chi^{\prime}}{4} C\left[\frac{\chi}{4}\right]
$$

with

$$
\begin{aligned}
C\left[\frac{\chi}{4}\right] \equiv & {\left[(\operatorname{Tr} \Im[f])^{\prime}\left(\frac{\chi}{4}\right)+\frac{1}{2} \operatorname{Tr}\left[(\Im[f])^{\prime}\left(\frac{\chi}{4}\right)(\Re[f])\left(\frac{\chi}{4}\right)\right]\right.} \\
& \left.-\frac{1}{2} \operatorname{Tr}\left[(\Im[f])\left(\frac{\chi}{4}\right)(\Re[f])^{\prime}\left(\frac{\chi}{4}\right)\right]\right],
\end{aligned}
$$

where $\chi^{\prime}$ denotes the first derivative with respect to $\left|\vec{z}_{T}\right|$, and $(\Im[f])^{\prime}$ and $(\Re[f])^{\prime}$ are the first derivatives of the real and imaginary parts of the color function $f$. Inserting (18) into (21) yields the lensing function in an Abelian $U(1)$-theory

$$
\mathcal{I}_{U(1)}^{i}\left(x, \vec{b}_{T}\right)=(1-x) \frac{b_{T}^{i}}{4\left|\vec{b}_{T}\right|} \chi^{\prime}\left(\frac{\left|\vec{b}_{T}\right|}{1-x}\right)\left(1+\cos \chi\left(\frac{\left|\vec{b}_{T}\right|}{1-x}\right)\right) .
$$

Likewise by using (19) the lensing function in an $S U(2)$-theory is given by

$$
\mathcal{I}_{S U(2)}^{i}\left(x, \vec{b}_{T}\right)=\frac{(1-x) b_{T}^{i}}{16\left|\vec{b}_{T}\right|} \chi^{\prime}\left(3\left(1+\cos \frac{\chi}{4}\right)+\left(\frac{\chi}{4}\right)^{2}-\sin \frac{\chi}{4}\left(\frac{\chi}{4}-\sin \frac{\chi}{4}\right)\right),
$$

where $\chi=\chi\left(\frac{\left|\vec{b}_{T}\right|}{1-x}\right)$. In an $S U(3)$-theory, we use the approximate color function $f$ to calculate the lensing function as a function of the eikonal amplitude. In Fig. 2 the function $C\left[\frac{\chi}{4}\right]$ is plotted versus $\frac{\chi}{4}$ for various approximations. While the convergence of the power series seems to be better for $S U(2)$ than in the $S U(3)$ case where the numerical result calculated with eight coefficients agrees with the analytical result up to $\frac{\chi}{4} \sim 2$, we can trust the numerical result computed with eight coefficients up to $\frac{\chi}{4} \sim 1.5$ for $S U(3)$.

In order to numerically estimate the lensing function and in turn the Boer Mulders function we seek to utilize the infrared behavior of the gluon and the running coupling in the non-perturbative regime where we infer that the soft gluon transverse momentum defines the scale at which the coupling is evaluated. These two quantities have been extensively studied in the infrared limit in the DysonSchwinger framework ${ }^{73}$ and in lattice QCD. ${ }^{74}$ We use calculations of these quantities from Dyson-Schwinger equations ${ }^{73}$ where both $\alpha_{s}$ and $\mathcal{D}^{-1}$ are defined in the infrared limit (details can be found in a forthcoming publication). This determines the eikonal phase and thus the lensing functions (21) for a $U(1), S U(2)$ and $S U(3)$ color function. We plot the results in Fig. 3 for a color function for $U(1), S U(2)$, $S U(3)$. While we observe that all lensing functions are attractive and fall off at large transverse distances, they are very different in size at small distances.

Using the eikonal model for the lensing function together with the spectator model for the GPD $H_{1}^{\pi}$ we present predictions of the relation (4) for the first moment of the pion Boer-Mulders function $h_{1}^{\perp(1)}$. We fix the six free model parameters $m_{s}$, 

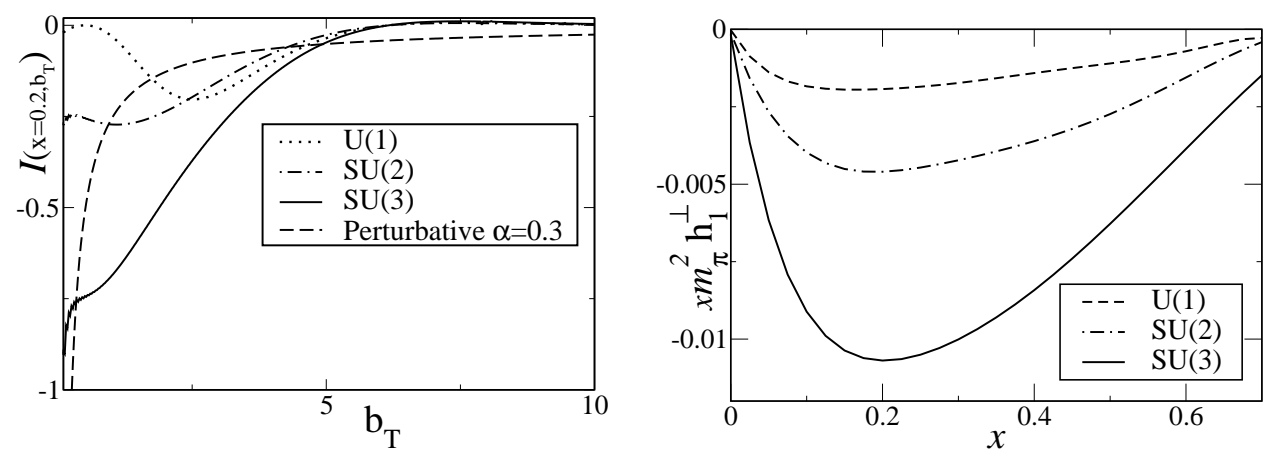

Fig. 3. Left: The lensing function $\mathcal{I}^{i}\left(x, \vec{b}_{T}\right)$ from Eq. (21) for $U(1), S U(2)$ and $S U(3)$ for $x=0.2$ at a scale $\Lambda_{Q C D}=0.2 \mathrm{GeV}$. For comparison we also plot the perturbative result with an arbitrary value for the coupling, $\alpha=0.3$. Right: First moments of the pion Boer-Mulders function calculated by means of the relation to the chirally-odd GPD $\mathcal{H}_{1}^{\pi}$ for a $S U(3), S U(2)$, and $U(1)$ gauge theory.

$m_{q}, \Lambda, \lambda, g_{\pi}$ and $n$ in (10) that we need to determine by fitting to pion data. In order to do so we determine the chiral-even GPD $F_{1}^{\pi}$ in the spectator model by investigating different limits of $F_{1}^{\pi}$. When integrated over $x$, the GPD reduces to the pion form factor $F^{\pi^{+}}\left(Q^{2}\right)=-F^{\pi^{-}}\left(Q^{2}\right)$. An experimental fit of the Pion form factor to data is presented in Refs. 75,76 , and up to $Q^{2}=2.45 \mathrm{GeV}^{2}$ a reasonable fit to the data is displayed by the monopole formula $F_{\text {fit }}\left(Q^{2}\right)=\left(1+1.85 Q^{2}\right)^{-1}$. This procedure is expected to predict the $t$-dependence of the chirally-odd GPD $H_{1}^{\pi}$ reasonably well up to $Q^{2}=2.45 \mathrm{GeV}^{2}$. In order to fix the $x$-dependence of $H_{1}^{\pi}$ we fit the collinear limit $F_{1}^{\pi}(x, 0,0)$ to the valence quark distribution in a pion, $v(x)$. A parameterization for this object was given for example by GRV in Ref. 77 at a scale $\mu^{2}=2 \mathrm{GeV}^{2}$. Reasonable agreements of the Form Factor- $F_{1}^{\pi}(x, 0,0)$ with the data fits were obtained for the parameters $m_{q}=0.834 \mathrm{GeV}, m_{s}=0.632 \mathrm{GeV}$, $\Lambda=0.067 \mathrm{GeV}, \lambda=0.448 \mathrm{GeV}, n=0.971, g_{\pi}=3.604$. With the predicted GPD $H_{1}^{\pi}$ and the lensing function $\mathcal{I}^{i}\left(x, \vec{b}_{T}\right)$ as input we can use the relation (4) to give a prediction for the valence contribution to the first $k_{T}$-moment of the pion BoerMulders function, which we can write as,

$$
m_{\pi}^{2} h_{1}^{\perp(1)}(x)=2 \pi \int_{0}^{\infty} d b_{T} b_{T}^{2} \mathcal{I}\left(x, b_{T}\right) \frac{\partial}{\partial b_{T}^{2}} \mathcal{H}_{1}^{\pi}\left(x, b_{T}^{2}\right) .
$$

We present numerical results for $x m_{\pi}^{2} h_{1}^{\perp(1)}(x)$ shown in Fig. 3 for a $U(1), S U(2)$ and $S U(3)$ gauge theory. ${ }^{42}$ It was argued in Ref. 57 that a negative sign of the lensing functions indicates attractive FSIs. We find that the lensing function is negative for the both the Abelian and non-Abelian gauge theories. The magnitude of the $S U(3)$ result is about 0.01 , while the $S U(2)$ result and $U(1)$ result are smaller. One observes a growth of the pion Boer-Mulders function with $N_{c}$ which was also predicted by a model-independent large $N_{c}$ analysis. ${ }^{78}$ So far the pion Boer-Mulders function is unknown but maybe determined from a future pion-proton Drell-Yan experiment 
to be performed at COMPASS. Once a pion Boer-Mulders is extracted our analysis can be used to verify quantitatively GPD - TMD relations. An extraction of the other T-odd parton distribution, the proton Sivers function $f_{1 T}^{\perp(1)}$, from SIDIS data measured at HERMES and COMPASS reveals an effect of the magnitude of about 0.04, four times larger than our prediction. A similar calculation for the proton Sivers function will be reported elsewhere.

\section{Acknowledgments}

L.G. dedicates this paper to Anatoli Efremov on the occasion of his 75th birthday. I am grateful to the organizers of the workshop Recent Advances in Perturbative QCD and Hadronic Physics ECT ${ }^{\star}$, Trento (Italy) for their efforts which made this memorable event possible. L.G. acknowledges support from U.S. Department of Energy under contract DE-FG02-07ER41460. Notice: Authored by Jefferson Science Associates, LLC under U.S. DOE Contract No. DE-AC05-06OR23177. The U.S. Government retains a non-exclusive, paid-up, irrevocable, world-wide license to publish or reproduce this manuscript for U.S. Government purposes.

\section{References}

1. G. Bunce et al., Phys. Rev. Lett. 36, 1113 (1976).

2. W. H. Dragoset et al., Phys. Rev. D18, 3939 (1978).

3. J. Antille et al., Phys. Lett. B94, 523 (1980).

4. FNAL-E704, D. L. Adams et al., Phys. Lett. B264, 462 (1991).

5. HERMES, A. Airapetian et al., Phys. Rev. Lett. 84, 4047 (2000), hep-ex/9910062.

6. HERMES, A. Airapetian et al., Phys. Rev. Lett. 94, 012002 (2005), hep-ex/0408013.

7. CLAS, H. Avakian et al., Phys. Rev. D69, 112004 (2004), hep-ex/0301005.

8. CLAS, H. Avakian, P. E. Bosted, V. Burkert, and L. Elouadrhiri, AIP Conf. Proc. 792, 945 (2005), nucl-ex/0509032.

9. COMPASS, C. Schill, Nucl. Phys. Proc. Suppl. 186, 74 (2009), arXiv:0809.2473.

10. STAR, J. Adams et al., Phys. Rev. Lett. 92, 171801 (2004), hep-ex/0310058.

11. PHENIX, S. S. Adler et al., Phys. Rev. Lett. 95, 202001 (2005), arXiv:hep-ex/0507073.

12. BRAHMS, I. Arsene et al., Phys. Rev. Lett. 101, 042001 (2008), arXiv:0801.1078.

13. STAR, B. I. Abelev et al., Phys. Rev. Lett. 101, 222001 (2008), arXiv:0801.2990.

14. G. L. Kane, J. Pumplin, and W. Repko, Phys. Rev. Lett. 41, 1689 (1978).

15. A. V. Efremov and O. V. Teryaev, Phys. Lett. B150, 383 (1985).

16. A. V. Efremov and O. V. Teryaev, Sov. J. Nucl. Phys. 36, 140 (1982).

17. J.-w. Qiu and G. Sterman, Phys. Rev. Lett. 67, 2264 (1991).

18. J.-w. Qiu and G. Sterman, Nucl. Phys. B378, 52 (1992).

19. J. P. Ralston and D. E. Soper, Nucl. Phys. B152, 109 (1979).

20. R. L. Jaffe and X.-D. Ji, Phys. Rev. Lett. 67, 552 (1991).

21. J. C. Collins, Nucl. Phys. B396, 161 (1993), hep-ph/9208213.

22. D. W. Sivers, Phys. Rev. D41, 83 (1990).

23. M. Anselmino, M. Boglione, and F. Murgia, Phys. Lett. B362, 164 (1995), arXiv:hep$\mathrm{ph} / 9503290$.

24. P. J. Mulders and R. D. Tangerman, Nucl. Phys. B461, 197 (1996), hep-ph/9510301.

25. D. Boer and P. J. Mulders, Phys. Rev. D57, 5780 (1998), hep-ph/9711485. 
26. D. Boer, P. J. Mulders, and F. Pijlman, Nucl. Phys. B667, 201 (2003), hep$\mathrm{ph} / 0303034$.

27. X. Ji, J.-W. Qiu, W. Vogelsang, and F. Yuan, Phys. Rev. Lett. 97, 082002 (2006), arXiv:hep-ph/0602239.

28. X. Ji, J.-W. Qiu, W. Vogelsang, and F. Yuan, Phys. Lett. B638, 178 (2006), arXiv:hep$\mathrm{ph} / 0604128$.

29. A. Bacchetta, D. Boer, M. Diehl, and P. J. Mulders, JHEP 08, 023 (2008), arXiv:0803.0227.

30. X.-d. Ji, J.-p. Ma, and F. Yuan, Phys. Rev. D71, 034005 (2005), arXiv:hepph/0404183.

31. D. W. Sivers, Phys. Rev. D43, 261 (1991).

32. S. J. Brodsky, D. S. Hwang, and I. Schmidt, Phys. Lett. B530, 99 (2002), hep$\mathrm{ph} / 0201296$

33. X.-d. Ji and F. Yuan, Phys. Lett. B543, 66 (2002), hep-ph/0206057.

34. G. R. Goldstein and L. Gamberg, (2002), Transversity and meson photoproduction, Proceedings of ICHEP 2002; North Holland, Amsterdam, p. 452 (2003), hepph/0209085, Published in Amsterdam ICHEP 452-454.

35. D. Boer, S. J. Brodsky, and D. S. Hwang, Phys. Rev. D67, 054003 (2003), hep$\mathrm{ph} / 0211110$.

36. L. P. Gamberg, G. R. Goldstein, and K. A. Oganessyan, Phys. Rev. D67, 071504 (2003), hep-ph/0301018.

37. L. P. Gamberg, G. R. Goldstein, and K. A. Oganessyan, Phys. Rev. D68, 051501 (2003), hep-ph/0307139.

38. A. Bacchetta, A. Schaefer, and J.-J. Yang, Phys. Lett. B578, 109 (2004), hep$\mathrm{ph} / 0309246$.

39. Z. Lu and B.-Q. Ma, Phys. Rev. D70, 094044 (2004), arXiv:hep-ph/0411043.

40. L. P. Gamberg, G. R. Goldstein, and M. Schlegel, Phys. Rev. D77, 094016 (2008), arXiv:0708.0324.

41. A. Bacchetta, F. Conti, and M. Radici, Phys. Rev. D78, 074010 (2008), arXiv:0807.0323.

42. L. Gamberg and M. Schlegel, (2009), arXiv:0911.1964, To appear in Phys. Lett. B.

43. M. Burkardt, Int. J. Mod. Phys. A18, 173 (2003), arXiv:hep-ph/0207047.

44. M. Burkardt and D. S. Hwang, Phys. Rev. D69, 074032 (2004), hep-ph/0309072.

45. S. Meissner, A. Metz, and K. Goeke, Phys. Rev. D76, 034002 (2007), arXiv:hep$\mathrm{ph} / 0703176$.

46. A. V. Belitsky, X.-d. Ji, and F. Yuan, Phys. Rev. D69, 074014 (2004), arXiv:hep$\mathrm{ph} / 0307383$.

47. S. Meissner, A. Metz, M. Schlegel, and K. Goeke, JHEP 08, 038 (2008), arXiv:0805.3165.

48. QCDSF, D. Brommel et al., Phys. Rev. Lett. 101, 122001 (2008), arXiv:0708.2249.

49. K. Goeke, A. Metz, and M. Schlegel, Phys. Lett. B618, 90 (2005), hep-ph/0504130.

50. J. C. Collins, Phys. Lett. B536, 43 (2002), hep-ph/0204004.

51. A. Bacchetta et al., JHEP 02, 093 (2007), hep-ph/0611265.

52. X.-d. Ji, J.-P. Ma, and F. Yuan, Phys. Lett. B597, 299 (2004), hep-ph/0405085.

53. J. C. Collins and A. Metz, Phys. Rev. Lett. 93, 252001 (2004), hep-ph/0408249.

54. R. D. Tangerman and P. J. Mulders, (1994), hep-ph/9408305.

55. D. Boer, Phys. Rev. D60, 014012 (1999), hep-ph/9902255.

56. S. Arnold, A. Metz, and M. Schlegel, Phys. Rev. D79, 034005 (2009), arXiv:0809.2262.

57. M. Burkardt, Nucl. Phys. A735, 185 (2004), hep-ph/0302144.

58. M. Diehl, Phys. Rept. 388, 41 (2003), hep-ph/0307382. 
59. K. Goeke, M. V. Polyakov, and M. Vanderhaeghen, Prog. Part. Nucl. Phys. 47, 401 (2001), hep-ph/0106012.

60. A. V. Belitsky and A. V. Radyushkin, Phys. Rept. 418, 1 (2005), hep-ph/0504030.

61. M. Burkardt, Phys. Rev. D72, 094020 (2005), hep-ph/0505189.

62. M. Diehl and P. Hagler, Eur. Phys. J. C44, 87 (2005), hep-ph/0504175.

63. Z. Lu and I. Schmidt, Phys. Rev. D75, 073008 (2007), hep-ph/0611158.

64. S. Meissner, A. Metz, and M. Schlegel, JHEP 08, 056 (2009), arXiv:0906.5323.

65. H. D. I. Abarbanel and C. Itzykson, Phys. Rev. Lett. 23, 53 (1969).

66. H. M. Fried, Y. Gabellini, and J. Avan, Eur. Phys. J. C13, 699 (2000).

67. R. Jakob, P. J. Mulders, and J. Rodrigues, Nucl. Phys. A626, 937 (1997), hep$\mathrm{ph} / 9704335$.

68. H. M. Fried, Gif-sur-Yvette, France: Ed. Frontieres (1990) 326 p.

69. F. Bloch and A. Nordsieck, Phys. Rev. 52, 54 (1937).

70. L. P. Gamberg, D. S. Hwang, A. Metz, and M. Schlegel, Phys. Lett. B639, 508 (2006), hep-ph/0604022.

71. H. M. Fried and Y. Gabellini, Phys. Rev. D55, 2430 (1997), arXiv:hep-ph/9607270.

72. H. M. Fried, Y. Gabellini, T. Grandou, and Y. M. Sheu, (2009), arXiv:0903.2644.

73. R. Alkofer, C. S. Fischer, F. J. Llanes-Estrada, and K. Schwenzer, Annals Phys. 324, 106 (2009), arXiv:0804.3042.

74. A. Sternbeck and L. von Smekal, (2008), arXiv:0811.4300.

75. Jefferson Lab, H. P. Blok et al., Phys. Rev. C78, 045202 (2008), arXiv:0809.3161.

76. Jefferson Lab, G. M. Huber et al., Phys. Rev. C78, 045203 (2008), arXiv:0809.3052.

77. M. Gluck, E. Reya, and A. Vogt, Z. Phys. C53, 651 (1992).

78. P. V. Pobylitsa, (2003), hep-ph/0301236. 